\title{
Una centena luminosa: homenaje a Fernando Tola Mendoza.
}

Lía Rodríguez de la Vega ${ }^{1}$

El Dr. Fernando Tola Mendoza nació en Lima, Perú, el 20 de octubre de 1915. Realizó sus estudios secundarios en Bélgica (1930-1935), en la Sección Greco-Latina del Athénée Saint-Gillesde Bruxelles, y sus estudios universitarios en la Universidad Nacional Mayor de San Marcos, Lima, donde obtuvo los títulos de Bachiller (Licenciado) en Humanidades (1939), Doctor en Literatura (1939), Abogado (1944) y Profesor Emérito (1972).Trabajó allí con Ippolito Galante, profesor italiano con quien fundaría el Instituto Superior de Lingüística y Filología y dictó cursos de latín, griego y literatura griega y orientales (entre 1935 y 1964) y desde 1958, de sánscrito, pali, Filosofía de la India , Budismo e Hinduismo.

Llegado a Buenos Aires, iniciaría su tarea en la Facultad de Filosofía y Letras de la Universidad de Buenos Aires, dictando cursos de Sánscrito, Introducción a la Filosofía Hindú y Seminarios sobre Filosofía de la India, en la Sección de Estudios de Filosofía Oriental, del Departamento de Filosofía de dicha facultad, pasando luego por otras instituciones.

En 1989, el Dr. Tola Mendoza creó, junto a su esposa Carmen Dragonetti, un Instituto de Investigaciones, la Fundación Instituto de Estudios Budistas (FIEB), institución reconocida por el CONICET como lugar de trabajo para realizar investigaciones científicas, donde ambos investigadores llevan a cabo sus tareas, siendo Vice- Presidente y Presidente de la misma, respectivamente. La fundación desarrolla sus actividades académicas buscando "promover, abordar y llevar a cabo estudios e investigaciones sobre el Budismo, sobre las culturas en las cuales éste ha florecido (Corea, China, India, Japón, Tíbet, Sudeste Asiático, etc.) y sobre los idiomas por él utilizados (chino, japonés, pali, sánscrito, tibetano, etc.)" y cuenta con la mayor biblioteca especializada de Indología en el mundo de habla hispana. Se llevan a cabo allí cursos de nivel de posgrado dictados por los Dres. Tola y Dragonetti sobre las temáticas mencionadas.

En 1991 los doctores Tola y Dragonetti, junto con el editor Sergio Mondragón, crearon la Revista de Estudios Budistas (REB), primera revista de habla hispana en su género, que quedaría inaugurada en un acto llevado a cabo en El Colegio de México (junio de 1991), en el que se hizo la presentación del primer número. La revista produjo trece números entre los años 1991 y 1997, siendo editada en México y dirigida y programada por ellos desde Buenos Aires. Contó en su Comité Consultivo con los más prestigiosos

\footnotetext{
${ }^{1}$ Lía Rodríguez de la Vega es Experta en Hinduismo Yoga, Lic. en Estudios Orientales y Doctora en Relaciones Internacionales (USAL). Realizó una Estancia Postdoctoral en la Universidad de Rio Grande do Sul (Porto Alegra, Brasil). Es docente e investigadora de la Facultad de Ciencias Sociales de la Universidad de Palermo, de la Facultad de Ciencias Sociales de la Universidad Nacional de Lomas de Zamora, entre otras. Autora de diversas publicaciones, participó y participa de distintos eventos académicos.
} 
especialistas en Budismo del mundo, tales como Luis O. Gómez (Universidad de Michigan USA), André Bareau (Collège de France), B.K.Matilal (Universidad de Oxford), Katsumi Mimaki (Universidad de Kyoto), Tsugunari Kubo (Instituto Internacional de Estudios Budistas), Akira Yuyama, E. Steinkellner (Universidad de Viena) y L. Schmithausen (Universidad de Hamburgo). La publicación, académica y sin fines de lucro, es en la actualidad de acceso libre a través de internet.

El Dr. Tola Mendoza ocupó diversos cargos a lo largo de su carrera, entre los que se cuentan el de Fundador y Director del Instituto de Lenguas y Culturas Orientales, Fundador y Director de la Colección "Clásicos de Oriente", Director del Instituto de Filología, Director de la Revista Sphinx², Director del Departamento de Publicaciones, etc. (en la Facultad de Letras de la Universidad Nacional Mayor de San Marcos, Lima, Perú). Fue también Consejero Cultural en la Embajada del Perú en la India (1964 - 1969). Fue Vicepresidente y luego Presidente de la Sociedad Argentina de Orientalistas (SADO), Vicepresidente de la Associaçao Latinoamericana de Sanscritistas (ALAS), Fundador y Director del Seminario de Indología del Centro de Investigaciones Filosóficas (CIF), Vice-Presidente y Fundador de la Fundación Instituto de Estudios Budistas FIEB. Ha sido Investigador del Consejo Nacional de Investigaciones Científicas y Técnicas (CONICET) (Categoría: Investigador Superior), Overseas Research Fellow de The International Institute for Buddhist Studies/ IIBS, Tokyo, Japón y Overseas Reseach Fellow (Honorary) del Zaike Bukkyo Kokoro no Kenkyujo (Essential Lay Buddhism Study Center), Tokyo, Japón.

Ha recibido también numerosas distinciones, tales como la de Profesor Emérito de la Universidad Nacional Mayor de San Marcos (Lima, Perú, 1972), la "Orden del Sol", en el Grado de "Gran Oficial" (con la que fue condecorado por el gobierno de Perú, en 1984, por sus aportes a la Ciencia y la Cultura en el Perú y en la Argentina), las "Palmas Magisteriales" en el Grado de "Amauta" (condecorado por el gobierno de Perú, en 1986, por su contribución a la formación de discípulos tanto en Perú como en Argentina), Miembro correspondiente de la Academia Peruana de la lengua (1995- ), Overseas Research Fellow, de The International Institute for Buddhist Studies, Tokyo, Japón, 1990-, Miembro de Honor de la Sociedad Científica Argentina (2000), Miembro Honorario de la Sociedad Internacional de Estudios Budistas/IABS (2011), etc.

Ha obtenido también distintas becas a lo largo de su trayectoria profesional, entre las que puede mencionarse la Beca del Gobierno Italiano (1936), Beca de la UNESCO para Grecia (1955), Beca del Gobierno Francés (1962), Beca del Gobierno Alemán (1962), Beca de la UNESCO para Holanda y Checoslovaquia (1964), Beca de la UNESCO para la India (en 1964, a través de la cual trabajó en el Bhandarkar Oriental Research Institute de Poona -Puna-), Beca de Investigación del Institute for Advanced Studies of World

\footnotetext{
${ }^{2}$ Rodriguez Rea (1990) aporta valiosa información sobre esta revista, órgano del Instituto Superior de Filología y Lingüística de la Universidad Nacional Mayor de San Marcos.
} 
Religions, de The State University of New York at Stony Brook como Lawrence Research Foundation Research Fellow (enero a abril de 1989), Beca de Investigación del International Institute for Buddhist Studies de Tokyo, Japón (abril a octubre de 1989), etc.

Señalando siempre la ineludible necesidad de acceder a la versión en lengua original de los textos abordados, ha abarcado el estudio de distintos idiomas, como el sánscrito, sánscrito budistahíbrido, pali, chino, tibetano y japonés. De igual modo, maneja francés, inglés, alemán, portugués, italiano, español, hindi, griego, latín, persa antiguo, etc.

Su impresionante trayectoria profesional de gran parte de sus 100 años recién cumplidos, dan cuenta de su labor original en investigación científica, la formación de discípulos y la creación y dirección de centros de investigación, al tiempo que del reconocimiento que junto con su esposa han logrado en el ámbito internacional. Su vasta obra incluye entre muchos otros, el indispensable Filosofía de la India. Del Veda al Vedānta, el sistema Samkhya. El mito de la oposición entre "pensamiento" indio y "filosofía occidental", On the Myth of the Opposition between Indian Thought and Western Philosophy, On Voidness, Budismo. Unidad y Diversidad, Being as Consciousness. Yogacara Philosophy of Buddhism, El Sutra del Loto de la Verdadera Doctrina -traducción al español del texto sánscrito del Saddharmapundarıkasutra-, Ideología o Filosofía. El Nazismo, Erich Frauwallner y Martin Heidegger, etc ${ }^{3}$.

Rodriguez de la Vega y Vofchuk (2010) señalan que en el campo de la Indología, de los Estudios Budistas, de la Filosofía de la India y de la Filosofía Budista ambos investigadores han hecho una serie muy apreciada de contribuciones, entre ellas, su permanente e incesante tarea para enfrentar con éxito el prejuicio de la no existencia de filosofía en la India; su investigación sobre anaditva en las diversas formas de manifestación del pensamiento de la India, que posibilitó la comprensión de temáticas no consideradas bajo esta nueva perspectiva hasta ese momento; su estudio sobre la refutación del todo al margen de las partes en el Pandit Ashoka, que mereció su publicación en un instituto especializado del Japón; su trabajo con los Yogasutras de Patañjali, que señaló nuevas direcciones de estudio; su interpretación de la teoría de la vaciedad en la filosofía Madhyamaka, que fuera expresamente valorada por T.R.V. Murti, gran especialista indio en el tema; sus aportes a la cuestión de la autenticidad de las obras de Nagarjuna; el estudio sobre el Budismo a través de sus textos en pali, desarrollado en ocasión de la Tesis de Licenciatura de la Dra. Dragonetti, trabajo pionero en el mundo de habla hispana; el estudio del Hastavalanamaprakarana (texto tibetano) de Aryadeva, sus estudios sobre el conflicto del cambio en el Budismo, que relevaron fuentes no atendidas con anterioridad a su tarea y que los llevaron a sostener ideas contrarias a las sustentadas por especialistas

\footnotetext{
${ }^{3}$ Más información sobre su obra puede consultarse en Tripathi (2012).
} 
internacionales como Hubert Durt, discípulo de Etienne Lamotte, y editor de la revista Cahiers, en la cual se publicarían los resultados de tal investigación; etc.

En el ámbito filológico han realizado aportes de gran importancia como las primeras ediciones críticas del Avayavinirakarana de Pandit Ashokay Catustava de Nagarjuna (textos sánscritos), Alambanaparikshade Dignaga, Vaidalyaprakarana de Nagarjuna, Shunyatasaptati de Nagarjuna, Bhavasankrantisutra (textos tibetanos), etc., además de otras ediciones publicadas sobre otros textos que, aunque no son las primeras traducciones, son las más modernas, como el caso de Yuktishashtika, de Nagarjuna. En el caso particular de la traducción del Sutra del Loto, del sánscrito budista híbrido al español, realizada a lo largo de diez años, cabe señalar que coloca al español en un lugar de particular relevancia, atendiendo a que solamente existen dos traducciones realizadas en el siglo pasado, al inglés y francés.

A ese aporte original, que hace que Rivera Martínez (2008) se refiera a él como "un peruano universal", se suma el de haber conformado (y seguir haciéndolo), la mayor biblioteca especializada en Indología en el mundo de habla hispana, abierta a la consulta de quien lo solicite; la constitución del español como lengua de conocimiento en este campo de estudios y el despliegue, a lo largo de sus carreras, de una ética inquebrantable que no solamente reconoció la igualdad de enunciación de las distintas culturas sino que hizo de ello un valor que sería transmitido a todos los alumnos y discípulos que formaron (y forman), instruyendo en una práctica real del llamado diálogo entre culturas, anticipándose a los debates acerca de la multi/interculturalidad y la relación entre cultura y poder.

Por todo lo mencionado y lo mucho que excede estas páginas, saludo (en mi nombre personal y el de todos aquellos que hemos tenido el honor de ser sus alumnos) la primera luminosa centena del Dr. Fernando Tola Mendoza, con la esperanza de que su ejemplo no nos abandone jamás y su juventud inunde no solamente nuestra maravillosa memoria de él sino, y fundamentalmente, nuestras acciones, como personas, docentes e investigadores. 


\section{Referencias}

Rivera Martínez, E. (2008). El don de las lenguas: Fernando TolaMendoza. El Comercio, Lima (7 de septiembre). Recuperado de http://www1.uprh.edu/rsoto/Fernando Tola Mendoza.pdf

Rodriguez de la Vega, L. y Vofchuck, R. (2010) Los estudios sobre la India en la República Argentina. Homenaje a Fernando Tola y Carmen Dragonetti. Publicación realizada en ocasión del Congreso Nacional de ALADAA: “Asia y África en Argentina. Caminos hacia una sociedad intercultural”.20 y 21 de agosto de 2010. Escuela de Estudios Orientales, Universidad del Salvador. Auspiciado por ALADAA, Sección Argentina; cátedra de Demografía Social, Departamento de Derecho y Ciencia Política, UNLaM y cátedra de Sánscrito, Facultad de Filosofía y Letras, UBA.

Rodriguez Rea, M. A. (1990). Guía de la Revista Sphinx (1937-1967). BIRA, 15, 213-252. Recuperado de http://revistas.pucp.edu.pe/index.php/boletinira/article/viewFile/9517/9922

Tola, F. y Dragonetti, C. (2014). Ideología o Filosofía. El Nazismo, Erich Frauwallner y Martin Heidegger. Buenos Aires: Las Cuarenta.

(2008). Filosofía de la India (Del Veda al Vedānta, el sistema Samkhya). El mito de la oposición entre "pensamiento" indio y "filosofía" occidental. Barcelona: Kairós.

(2004). OntheMyth of theOppositionbetweenIndianThought and Western Philosophy.Hildesheim: OlmsVerlag. (2004). Budismo. Unidad y Diversidad. Carmel, NY, USA: TheBuddhistAssociation of theUnitedStates.

(2004). Being as Consciousness. YogacaraPhilosophy of Buddhism. Delhi: MotilalBanarsidass. (2002). OnVoidness. Delhi: MotilalBanarsidass. (2nd edition). (1999). El Sutra del Loto de la Verdadera Doctrina. México: El Colegio de México. 
Tripathi, R. (Ed.) (2012). SixtyYears of SanskritStudies (1950-2010).Vol. 2: Countriesotherthan India. New Delhi: RashtriyaSanskritSansthan(undertheMinistry of Human ResourceDevelopment,Government of India). 